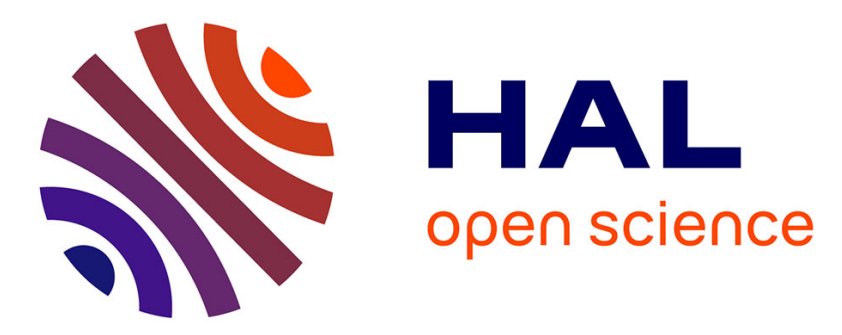

\title{
Tumor angiogenesis assessment using multi-fluorescent scans on murine slices by Markov Random Field framework
}

\author{
Oumeima Laifa, Delphine Le Guillou-Buffello, Daniel Racoceanu
}

\section{- To cite this version:}

Oumeima Laifa, Delphine Le Guillou-Buffello, Daniel Racoceanu. Tumor angiogenesis assessment using multi-fluorescent scans on murine slices by Markov Random Field framework. SIPAIM, 2017, San Andres Island - Colombia, Colombia. hal-01615267

\section{HAL Id: hal-01615267 \\ https://hal.science/hal-01615267}

Submitted on 12 Oct 2017

HAL is a multi-disciplinary open access archive for the deposit and dissemination of scientific research documents, whether they are published or not. The documents may come from teaching and research institutions in France or abroad, or from public or private research centers.
L'archive ouverte pluridisciplinaire HAL, est destinée au dépôt et à la diffusion de documents scientifiques de niveau recherche, publiés ou non, émanant des établissements d'enseignement et de recherche français ou étrangers, des laboratoires publics ou privés. 


\title{
Tumor angiogenesis assessment using multi-fluorescent scans on murine slices by Markov Random Field framework Oumeima. Laifa*ac ${ }^{\text {ac }}$, Delphine. Le Guillou-Buffello ${ }^{\mathrm{a}}$, Daniel Racoceanu ${ }^{\text {ab }}$ \\ a Sorbonne Universités, UPMC Univ Paris 06, CNRS, INSERM, Laboratoire d'Imagerie Biomédicale, Paris, France; 'Pontifícia Universidad Católica Del Perú,1801 Av. Universitaria San Miguel, Lima32; \\ ${ }^{c}$ Bioinformatics institute, 30 Biopolis Street, Singapore 138671
}

\begin{abstract}
The fundamental role of vascular supply in tumor growth makes the evaluation of the angiogenesis crucial in assessing effect of anti-angiogenic therapies. Since many years, such therapies are designed to inhibit the vascular endothelial growth factor (VEGF). To contribute to the assessment of anti-angiogenic agent (Pazopanib) effect on vascular and cellular structures, we acquired data from tumors extracted from a murine tumor model using Multi-Fluorescence Scanning. In this paper, we implemented an unsupervised algorithm combining the Watershed segmentation and Markov Random Field model (MRF). This algorithm allowed us to quantify the proportion of apoptotic endothelial cells and to generate maps according to cell density. Stronger association between apoptosis and endothelial cells was revealed in the tumors receiving anti-angiogenic therapy $(n=4)$ as compared to those receiving placebo $(n=4)$. The percentage of apoptotic cells on tumor area is mostly endothelial. Lower density cells were detected in tumor slices presenting higher apoptotic endothelial areas.
\end{abstract}

Keywords: Anti-angiogenic, apoptosis, watershed, multi-fluorescence images, MRF, pazopanib.

\section{INTRODUCTION}

The growth of tumors is strongly related to neovascularization via angiogenic process. During this process, tumor cells secrete growth factors that activate the proliferation and migration of endothelial cells and allow capillary morphogenesis. Hypoxic tumor regions remain despite this neovascularization. Hypoxic zones continue to stimulate overproduction of vascular endothelial growth factor, VEGF $^{1,2}$.

Anti-angiogenic therapies have been developed to block pro-angiogenic factor such as $\operatorname{VEGF}^{3,4,5}$. Pazopanib is an oral angiogenesis inhibitor targeting vascular endothelial growth factor receptor (VEGF-R) ${ }^{4,6}$. In Metastatic Renal Cell Carcinoma (mRcc), single agent Pazopanib compared with placebo was shown to extend Progression Free Survival (PFS) in Sternberg and collaborator's work ${ }^{4,6}$.

However, VEGF inhibition is not effective in all cancer ${ }^{4}$. Therefore, we need more understanding on how the neoangiogenic vasculature can be effectively targeted in tumors ${ }^{5}$.

In this paper, we collected Multi-Fluorescence whole-slide images in a tumor model under anti-angiogenic Pazopanib therapy to develop and test techniques quantitatively assess the cell density, apoptosis and endothelial cells. Watershed segmentation was applied to evaluate the number of cells throughout the tumor sections. Stochastic image processing methods were used because of undefined structure of blood vessels and apoptotic cells in the image of our database.

Quantitative evaluation of tumor structures with histopathological precision across the full tumor cross section may ultimately contribute to the identification of architectural features that are often associated or predictive of therapeutic 
response $^{4}$. Data can also serve as valuable reference information as noninvasive imaging techniques are developed to probe the tumor environment during screening and therapy.

Several recent studies have shown the interest of fluorescent microscopy imaging segmentation ${ }^{7,8}$. This kind of imaging can be an important tool which allows better reproducibility than non-fluorescent immunostaining.

\section{DATA}

\subsection{Cell culture and tumor model}

Murine colorectal tumor cells, CT26 (American Type Culture Collection, Virginia) were cultured in a specific medium. Two thousand tumor cells in $100 \mu \mathrm{L}$ of $2.10^{6} \mathrm{cells} / \mathrm{ml}$ were then injected subcutaneously on the left flanks of $3 \mathrm{Balb} / \mathrm{c}$ mice to generate first generation tumors: G1. 16 days after cell implantation, we consider that tumors are large enough to be used to make second generation tumors: G2. Therefore, tumors were removed after euthanasia of the mice. Excised tumors were dissected into 20-40 $\mathrm{mm}^{3}$ fragments which were then implanted in the left flanks of eight other Balb /c mice $(\mathrm{n}=8)$ via a small incision that was closed with one or two sutures. The date of fragment implantation is referred to as day zero (D0).

We underline the need to make G2 tumors because there are more reproducible.

Thereafter, ultrasound imaging was performed every 2 to 3 days to evaluate the tumor size evolution. On Day 7, four mice, AA1, AA2, AA3 and AA4 were treated by gavage with anti-angiogenic drug, Pazopanib, repeated day by day (2 mg diluted in $100 \mu \mathrm{L}$ VOTRIENT ${ }^{\circledR}$ ). Mice labeled P1, P2, P3 and P4, received placebo by the same route and administration schedule. On Day 18, all mice were euthanized and tumors were removed carefully to conserve orientation relative to the anterior surface (skin side) and the longitudinal axis. Tumors were prepared for cryoconservation and embedded in Optimum Cutting Temperature gel (tissue-tek ® OCT, VWR, France).

Tumors were stored in a freezer at $-80^{\circ} \mathrm{C}$ until labeling for immunohistochemistry. All immunohistochemistry marking was performed on the same day for the entire sample set. Tumors were sectioned to obtain a central section along the longitudinal tumor axis. Sections were 8 to 10 microns thick.

\subsection{Immunohistochemistry labeling}

Triple immunohistochemistry marking was performed on each of the 8 slices ( 1 slice per mouse). Triple labeling provides marking of the neovascularization, apoptosis and total nuclei across the tumor surface such that the relative position of structures can be evaluated. Isolectin B4-A488 was used to mark endothelial cell showing neovascularization area. Caspase 3 IgG antibodies followed by a second anti-IgG antibody TRITC were used to mark apoptosis. 4', 6-diamidino-2phénylindole (DAPI) was used to mark nuclei.

\subsection{Automated multi-fluorescence scan imaging}

After labeling tumor slices, digital pathology data was obtained using a 2D scanner, Zeiss Axioscan (Zeiss, France). It allows faster acquisition more than any fluorescence microscopy. The scan is made field by field and assembled the high resolution fields into a whole slice image. The system can record data for up to 3 separate labels by filtering for the fluorophore of each marker. The RGB images obtained were recorded as CZI files and visualized using the image processing software ZEN. Example slices are presented in figure 1. Different structures of the tumor can be visualized separately. 


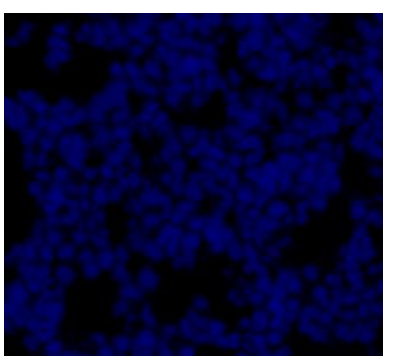

a

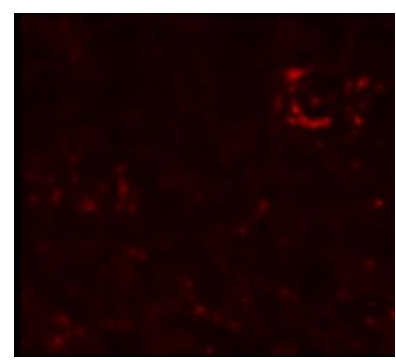

b

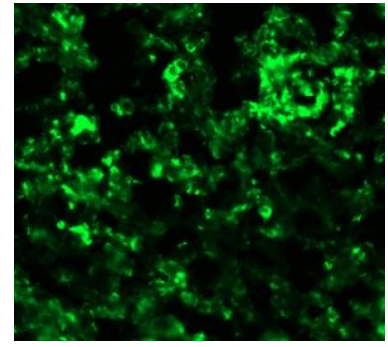

$\mathrm{C}$

Figure 1: One field (1024*1024 pixels) of the whole slice Multi-Fluorescence Scan imaging of tumor slice of P1 mouse. (a) DAPI channel representing nuclei in the field (b) Cyx channel representing apoptotic areas in the field (c) Cyc representing endothelial cells in the field (Magnification level: 215\%)

\section{METHODOLOGY}

\subsection{Image processing}

Quantification software was developed in house Matlab (version city) to evaluate number and density of structures based on the fluorescent microscope data. However, CZI files are not a supported format in Matlab. Thus, files were exported in Tagged Image Filed Format (TIFF) to preserve the best image quality possible. Our algorithm starts by obtaining 3 binary masks of images for each of the 8 tumor sections. 8905 Regions of interests (ROIs) were extracted at $0.325 \mathrm{um} /$ pixel resolution and stored in Tiles TIFF files with tiles size $(1024 * 1024$ pixels). This tile size was fixed by our team biologist. For each tumor's slice, segmentation was performed on each tile (1024*1024) separately. To contribute to the assessment of Pazopanib anti-angiogenic effect on vascular and cellular structures, quantification of the spatial distribution of nuclei cell, apoptotic area and neovascularization apoptotic area (endothelial cell) was required. We aim to get 3 binary masks of every tumor's slice:

- Nuclei segmentation, apoptotic area segmentation and apoptotic neovascularization

For each tumor slice, trained biologist has manually annotated the ground truth on the tiles using Aperio Imagescope for final evaluation.

\subsection{Nuclei density maps:}

In the literature $9,10,11$, one usually use a threshold method to separate the background from pixels belonging to object of interest. In our case, each $n$ connected group of pixels tagged as nuclei is analyzed, and then watershed segmentation was performed inside each component to split it into individual nuclei. Watershed segmentation method was applied to the blue images describing the nuclei distribution. This method provided a binary mask of all nuclei in the tumor area as shown in figure 2. These segmentation tools are derivate from mathematical morphology. Watershed segmentation allows a partition into multiple regions according to a topographic approach ${ }^{12}$.

Then, by calculating the number of nucleus on the mask in each $(1024 * 1024)$ pixel tile, we obtained maps describing the local density (nucleus number per tile/tile area) and distribution of nuclei. In fact, the local density per each tile zone on the image was quantified on the mask following equation (1) and grouped into a matrix called the nuclei matrix.

$$
N D_{\text {tile }}=\frac{N N_{\text {tile }}}{T A_{\text {tile }}}
$$


Where ND is the nuclei density per tile, NN is nuclei number through tile area and TA is tile area. Based on the cell structure, maps of the density of nuclei were calculated across each whole slide image.

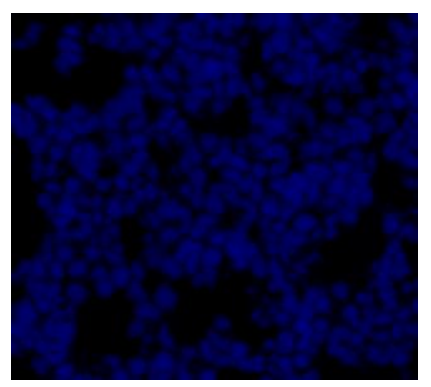

a

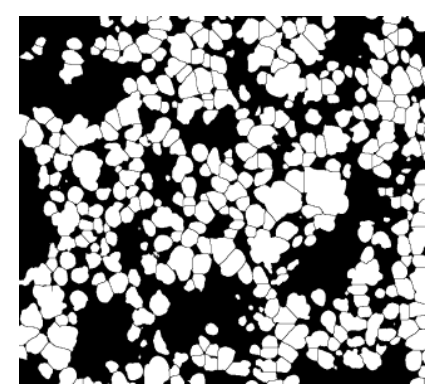

b

Figure 2: Watershed segmentation of DAPI imaging (a) DAPI image P1 mouse placebo (b) mask of nuclei through one tile tumor area

\subsection{Apoptotic endothelial cell assessment:}

The structure of endothelial cells and apoptotic regions are not well defined as shown in figure1 (b) and (c), respectively. A global threshold was not sufficient to separate these regions from the background as shown in figure 3(b). Especially, there are fluorophore artifacts shown in figure3 (a) with dark green. Not all green area on the figure 3(a) represents endothelial cells. We thus chose to apply a stochastic segmentation method to evaluate these features. We used Markov random field model segmentation (MRFs) based on pixel intensity on mono-channel: green one for endothelial cells and red one for apoptotic area. We considered segmentation as a pixel labeling task to obtain an endothelial cell binary mask and a binary mask of apoptotic region throughout the tumor's cross-section. We aimed to classifie pixels into two classes. For each tumor's slice, segmentation was performed on each tile $(1024 * 1024)$ separately.

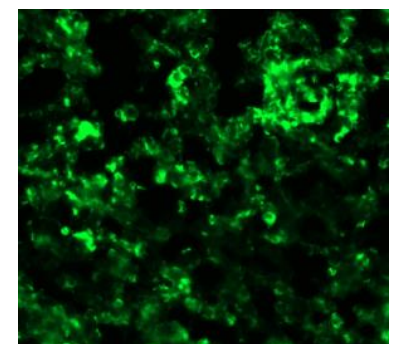

a

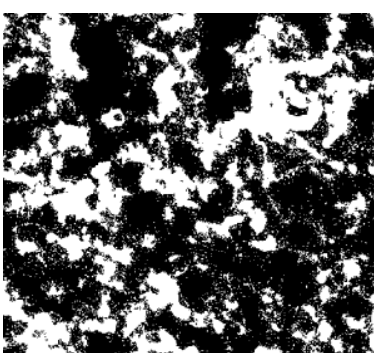

b

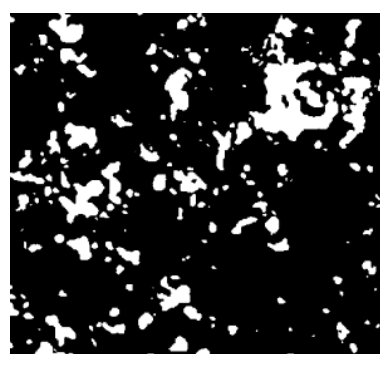

c

Figure 3: (a) endothelial cells in one tile (b) mask with threshold segmentation (c) mask with MRFs

Our goal is to segment each tile on 2 classes: background and endothelial cell. Our approach starts with a Gaussian Mixture $\operatorname{Model}^{13}$ (GMM) which segments the images on 3 classes: endothelial cell class, background and transition class pixel then a Markov Random Field (MRF) is applied to have the 2 classes. The third class represents uncertainty pixels between endothelial cell and artifacts. The main difficulty is that the model and its parameters are not known and need to be computed.

Our image $\mathrm{Y}$ is a set of intensity data $Y=\left\{y_{s}\right\}$ where $s$ is the location of a pixel $y$ in the image and $y_{s}$ its intensity. 
Let us note $X=\left\{x_{s}\right\}$ the segmented version of $Y=\left\{y_{s}\right\}$ where $x_{s} \in\{1,2, \ldots, K\}$ and $\mathrm{K}$ is the number of different regions. The pixel intensity $y_{s}$ belongs to a region $x_{s}$. Thanks to Bayes theorem shown in equation $(2), P(Y)$ is constant representing the probability of pixels intensity in the image. The conditional probability is then shown in equation (3).

$$
\begin{gathered}
P(X \mid Y)=\frac{P(Y \mid X) P(X)}{P(Y)} \\
P(X \mid Y) \alpha P(Y \mid X) P(X)
\end{gathered}
$$

$P(X)$ represents prior probability of one region $x_{s}$ and $P(Y \mid X)$ is the conditional probability density function of $y_{s}$ given the image region $x_{s}$.

\subsection{The prior probability:}

The prior probability is modeled by a Markov Random Field (MRF). Local neighborhood between pixels is taken into account. For an s location of a pixel $y$ the conditional probability satisfy the following equation:

$$
p\left(X_{x} \mid X_{R}, s \notin R\right)=p\left(X_{s} \mid X_{R}, R \in N_{s}\right) \text { (4) }
$$

where $N_{s}$ is a neighborhood of region $\mathrm{s}$ and $\mathrm{r}$ the neighbor region. Hamersley-Clifford theorem shows that the probability of $\mathrm{X}$ is given by a Gibbs distribution as follows:

$$
p(X) \alpha \exp \left[-\sum_{c \in C} V_{c}(X)\right]
$$

A subset $C \subseteq Y$ is called a clique if every pair of pixels in this subset is neighbors. For each clique $\mathrm{C}$ in the image, we can assign a value $V_{c}(X)$ which is the clique potential of $\mathrm{C}$. This clique potential is defined by equation (6)

$$
V_{c}(X)= \begin{cases}-\gamma_{c}, & x_{s}=x_{R} \\ \gamma_{c} & \text { Otherwise }\end{cases}
$$

\subsection{The conditional probability:}

Here a Gaussian distribution $P(Y \mid X)$ is defined by $\hat{X}=\left(m_{s}, \sigma\right)$ the mean and standard deviation respectively. The aim is the estimate the unknown parameters that maximize the log-likelihood function shown by equation (7).

$$
X_{\text {max }}=\arg \max _{x}\{p(x \in X \mid y \in Y)\}
$$


To do so we use Iterated Conditional Modes (ICM) algorithm. 40 iterations were enough for ICM to reach stable segmentation. The ICM requires an initial segmentation. K-mean method was applied to initialize the ICM.
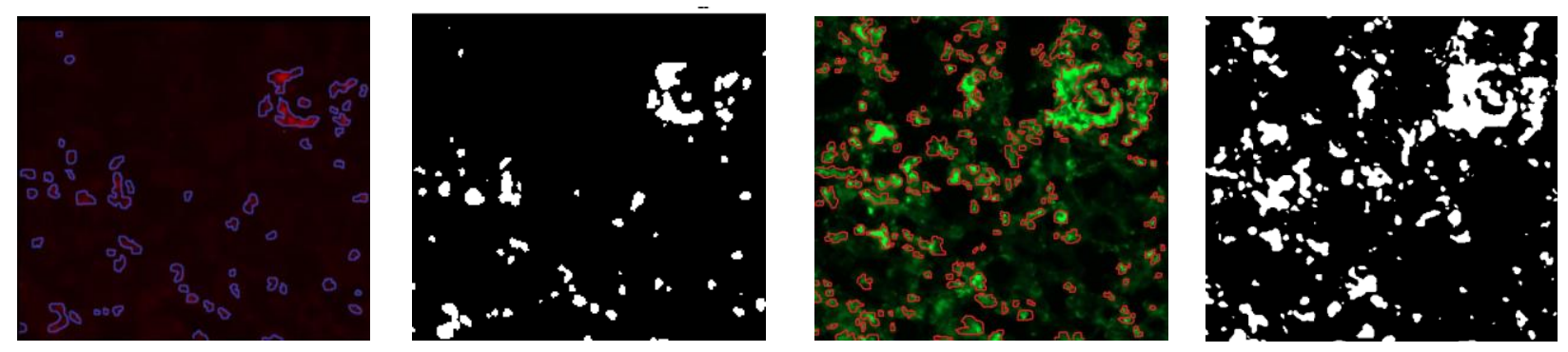

Figure 4: (a) ground truth of apoptotic cells through tumor area (b) binary mask of apoptotic area given by MRF segmentation (c) ground truth of endothelial cells through tumor area (d) binary mask of apoptotic area given by MRF segmentation.

Apoptotic tumor area was calculated by multiplying the binary mask of total cells by the binary mask of Nuclei. Endothelial apoptotic areas were calculated by multiplying the binary mask of endothelial cells by the mask of all apoptotic areas. One all these results are obtained we generated our cartographies and we calculate the percentage of endothelial apoptotic cell.

\section{Validation process:}

The F-score is used to measure the segmentation accuracy of masks obtained from our method: the apoptotic areas mask and the neovascularization mask. We consider a vascularization mask as true positive if it $50 \%$ area coincides with ground truth; otherwise it is considered false positive. The difference between the numbers of ground truth objects and number of truth positives is the number of false negative. Equation (6) defines F-score:

$$
\begin{gathered}
F 1 \text { score }=\frac{2 \text { precision } \text { recall }}{\text { precision }+ \text { recall }} \\
\text { Where precision }=\frac{T P}{T P+F P} \quad \text { and recall }=\frac{T P}{T P+F N}
\end{gathered}
$$

TP, FP and FN denote respectively the number of true positives, false positives and false negatives from all tiles given by the database.

\section{RESULTS}

The percentage of apoptotic endothelial cells on the whole slice tumor relative to the total number of apoptotic cells exceeded 50\% for all sections from the 4 mice treated with anti-angiogenic agent Pazopanib. Highest percentage (up to $70 \%$ ) was observed in tumor sections from AA1. The percentage of apoptotic endothelial cells in whole slices from mice receiving placebo did not exceed $40 \%$ as summarized in Figure 5. The percentage of apoptotic cells on tumor area is mostly endothelial except one aberration value considering P2. Our finding supports Kadambi's ${ }^{14}$ work showing that blocking vascular endothelial growth factor (VEGF) or its receptor (VEGFR2) leads to apoptosis of endothelial cells. 


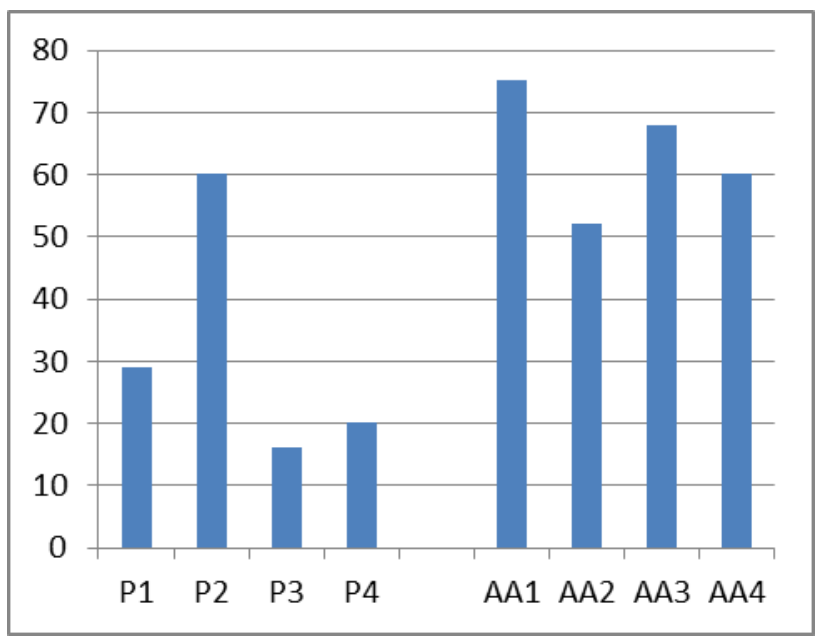

Figure 5: Percentage of apoptotic endothelial cells relative to the total apoptotic cells between placebos mice and mice treated by Pazopanib. P1, P2, P3 and P4 are mice receiving placebo. AA1, AA2, AA3 and AA4 are mice receiving pazopanib.

Maps of the density of nuclei cells were calculated across each whole slide image. Overall, the density values were higher for placebo mice as compared to mice receiving Pazopanib (figure 6). Regional variability of the cell density can also be observed on these maps. The lowest density cells (blue color on the map) were detected in tumor slices presenting the highest apoptotic endothelial areas (yellow color on the map).

Because of the few number of our database $(8$ mice), we cannot conclude about a correlation between the apoptotic neovascularization and the nuclei density. Thus, we highlight the importance of working on more slices. However, our result shows a specific relationship between apoptotic neovascularization and nucleus density in murine tumor treated by Pazopanib anti-angiogenic.

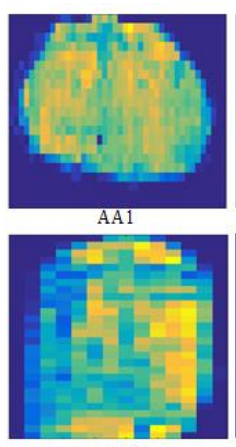

AA2
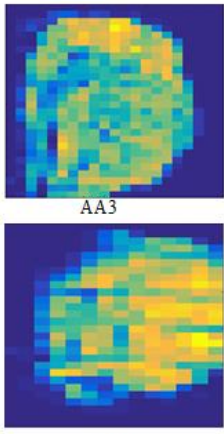

AA4
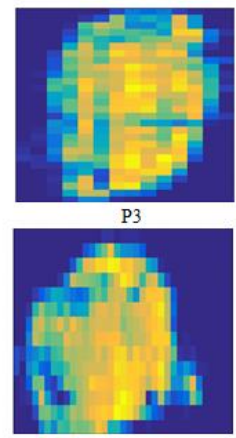

P1
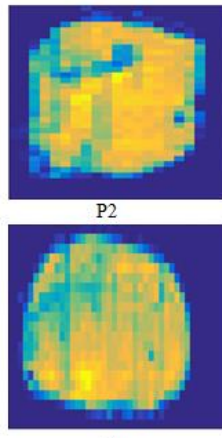

P4

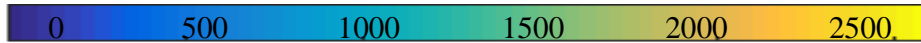

Figure 36 : Maps of cell density are displayed for a whole slice segment of from each mouse. The maps labeled $\mathrm{P}$ are from mice receiving placebo and those labeled AA from mice receiving anti-angiogenic agent. 
Results demonstrated that antiangiogenic therapy with Pazopanib generates apoptosis that is strongly associated with endothelial cells. Mapping of nuclei using a watershed segmentation method to separate different DAPI labeled cells on the tumor surface with an unsupervised method allowed us to establish homogeneous regions of cell density. Ultimately, this work will contribute to provide a high-resolution and full slide assessment of tumor modifications that can serve as reference measurements when validating non-invasive imaging techniques. Evaluation of our algorithm is shown in table1.

\begin{tabular}{|lcccccccc|}
\hline & precision & error & recall & error2 & accuracy & error3 & F-score & error4 \\
\hline P1 & 0.65 & 0.001 & 0.71 & 0.003 & 0.74 & 0.001 & 0.67 & 0.001 \\
\hline P2 & 0.71 & 0.003 & 0.72 & 0.001 & 0.69 & 0.003 & 0.71 & 0.002 \\
\hline P3 & 0.71 & 0.002 & 0.68 & 0.001 & 0.65 & 0.001 & 0.69 & 0.001 \\
\hline P4 & 0.69 & 0.001 & 0.72 & 0.002 & 0.73 & 0.002 & 0.70 & 0.002 \\
\hline AA1 & 0.68 & 0.002 & 0.69 & 0.003 & 0.71 & 0.001 & 0.67 & 0.001 \\
\hline AA2 & 0.71 & 0.001 & 0.72 & 0.001 & 0.70 & 0.001 & 0.71 & 0.001 \\
\hline AA3 & 0.66 & 0.001 & 0.71 & 0.001 & 0.69 & 0.002 & 0.67 & 0.001 \\
\hline AA4 & 0.77 & 0.002 & 0.65 & 0.003 & 0.65 & 0.002 & 0.70 & 0.003 \\
\hline
\end{tabular}

\section{CONCLUSION}

An experimental protocol as quantitative techniques were developed and applied to assess nuclei, apoptosis and endothelial cell distribution across whole-slice histological sections of tumors. Noninvasive imaging techniques were developed to probe the tumor environment during screening and therapy. We proposed watershed segmentation for the nuclei density quantification. GMM and MFR model was applied to resolve the endothelial cell and apoptotic areas segmentation problem.

During this work we faced two challenges. First, because the Zeiss scanner does not provide information about the Z dimension, thick or folded regions of tumor sections were not fully interpretable. To minimize folding, sections were cut as thinly and carefully as possible. Secondly, tears in the sections occurred in some cases. The need to work with thin sections exacerbated the risk of tearing. The level of bias in quantification resulting from tears and folds remains to be assessed in future work but this bias is not anticipated to be very strong based on the relatively limited surface area involved with such artifacts in our sections.

For individual patients, optimization of dose and schedule in anti-angiogenic therapies remains a challenge. Specific phenotypic changes to be quantified during anti-angiogenic therapy are needed. Biopsies can provide us this information however they are difficult to obtain. New imaging technology such as fluorescence imaging can serve as reference measurements as non-invasive and reproducible imaging techniques. 


\section{Acknowledgement}

The authors thank the CEF (Centre d'Explorations Fonctionnelles, Cordeliers' Research Center) for their technical support and help with animals care. We gratefully acknowledge support from the Fondation Recheche Médicale (Recherche soutenue par le FRM DBS20131128436). This work was performed, in part, on a platform of France Life Imaging network partly funded by the grant "ANR-11-INBS-0006". The authors would like to acknowledge Jérôme Griffon for preparation of tumor specimens.

\section{REFERENCES}

1. Harmey, J.H. and Bouchier-Hayes, D.,"Vascular endothelial growth Factor (VEGF), a survival factor for tumour cells: implications for anti-angiogenic therapy" SID Bioessays. Papers 24, 280-283 (2002).

2. Singh, Y., "Tumor Angiogenesis: Clinical Implications" SID Nepal Journal of Neuroscience. Papers 1, 61-63 (2004).

3. Jayson, G.C., Kerbel, R., Ellis, L.M. and Harris, A.L., "Antiangiogenic therapy in oncology : current status and future directions" SID Lancet. Papers 388, 518-529 (2016).

4. Vasudev, N.S.and Reynolds, A. R., "Anti-angiogenic therapy for cancer: current progress, unresolved questions and future directions" SID Angiogenesis. Papers 17, 471-494 (2014).

5. Mbeunkui, F., and Johann Jr, D. J., "Cancer and the tumor microenvironment: a review of an essential relationship" SID Cancer Chemother Pharmacol. Papers 63, 571-582(2009).

6. Sternberg, C.N., Davis, I.D., Mardiak, J., Szczylik, C., Lee, E., Wagstaff, J., Barrios, C.H., Salman, P., Gladkov, O.A., Kavina, A., Zarbá, J.J., Chen, M., McCann, L., Pandite, L., Roychowdhury, D.F. and Hawkins, R.E., " Pazopanib in locally advanced or metastatic renal cell carcinoma: Results of a randomized Phase 3 Trail" SID Jornal Of Clinical Oncology. Papers 28(6), 1061-1068 (2010).

7. Allalou, A. and Wählby, C., "BlobFinder, a tool for microscopy image cytometry" SID computer methods and programs in biomedicine. Papers 94, 58-65(2009).

8. Chen, X., Zhou, X., and Wong, S.T.C., "Automated segmentation, classification, and tracking of cancer cell nuclei in time-lapse microscopy" SID IEEE Transactions on biomedical engineering. Papers 53, 762-766 (2006).

9. Carpenter, A. E., Jones, T. R., Lamprecht, M.R., Clarke, C., Kang, I.H., Friman, O., Guertin, D.A, Chang, J.H., Lindquist, R. A., Moffat, J., Golland P., and Sabatini, D.M., "CellProfiler: image analysis software for identifying and quantifying cell phenotypes".SID Genome biology.Papers 7 (10), 100-111 (2006)

10. Al-amri, S.S., Kalyankar, N.V. and Khamitkar, S.D. " Image Segmentation by Using Threshold Techniques" SID journal of computing. Paper 2(5), 2151-9617(2010).

11. Osuna-Enciso, V., Cuevas, E. and Sossa, H."A comparison of nature inspired algorithms for multi-threshold image segmentation" SID Expert Systems with Applications. Paper 40(4), 1213-1219(2013)

12. Wang, D."A multiscale gradient algorithm for image segmentation using watershed"SID Pattern Recognition. Papers 30(12), 20432052 (1997).

13. Greenspan, H., Ruf, A and Goldberger, J."Constrained Gaussian mixture model framework for automatic segmentation of MR brain images" SID IEEE Transactions on Medical Imaging. Paper 25(9) (2006)

14. Kadambi, A., Mouta Carreira, C., Yun, CO., Padera, T.P., Dolmans, D.E., Carmeliet, P., Fukumura, D. and Jain, R.K. "Vascular endothelial growth factor (VEGF)-C differentially affects tumor vascular function and leukocyte recruitment: role of VEGF-receptor 2 and host VEGF-A" SID Cancer research. Papers 61, $2404-2408$ (2001). 